\title{
DOTATOC: a powerful new tool for receptor-mediated radionuclide therapy
}

\author{
A. Otte ${ }^{1}$, E. Jermann'1, M. Behe1, M. Goetze', H.C. Bucher ${ }^{2}$, H.W. Roser ${ }^{3}$, A. Heppeler ${ }^{1}$, J. Mueller-Brand1, \\ H.R. Maecke 1 \\ 1 Institute of Nuclear Medicine, University Hospital, School of Medicine, Basel, Switzeriand \\ 2 Department of Internal Medicine, University Hospital, School of Medicine, Basel, Switzerland \\ 3 Department of Radiological Physics, University Hospital, School of Medicine, Basel, Switzerland
}

Received 20 March and in revised form 4 April 1997

\begin{abstract}
This study presents the first successful use of a peptidic vector, DOTATOC, labelled with the $\beta$-emitting radioisotope yttrium-90, for the treatment of a patient with somatostatin receptor-positive abdominal metastases of a neuroendocrine carcinoma of unknown localization. Tumour response and symptomatic relief were achieved. In addition, the new substance DOTATOC was labelled with the diagnostic chemical analogue indium-111 and studied in three patients with histopathologically verified neuroendocrine abdominal tumours for its diagnostic sensitivity and compared with the commercially available OctreoScan. In all patients the kidney-to-tumour uptake ratio (in counts per pixel) was on average 1.9-fold lower with ${ }^{111}$ In-DOTATOC than with OctreoScan. DOTATOC could be a potential new diagnostic and therapeutic agent in the management of neuroendocrine tumours.
\end{abstract}

Key words: Somatostatin receptor-mediated internal radiotherapy - DTPA-D-Phe ${ }^{1}$-octreotide (OctreoScan) DOTA-D-Phe ${ }^{1}-\mathrm{Tyr}^{3}$-octreotide (DOTATOC) - Indium111 - Yttrium-90

Eur J Nucl Med (1997) 24:792-795

\section{Introduction}

In recent years the use of the radiolabelled somatostatin analogue ${ }^{11} 1$ In-DTPA-D-Phel-Octreotide (OctreoScan; DTPA: diethylene-triamine-penta-acetic acid) (Fig. 1) as a specific radiopharmaceutical for the in vivo detection of somatostatin receptor-positive tumours has been promulgated in clinical nuclear medicine, endocrinology and oncology $[1,2]$. Despite convincing diagnostic results, this analogue cannot be used for labelling with a

Correspondence to: A. Otte, Institute of Nuclear Medicine, University Hospital, School of Medicine, Petersgraben 4, CH-4031 Basel, Switzerland $\beta$-emitter such as yttrium-90 for therapy. Crucial for such a radiotracer is the development of a peptide chelator conjugate which can hold the radiometal with high stability in vivo in order to reduce haematopoietic toxicity due to bone marrow irradiation. Moreover, a hydrophilic conjugate with predominant kidney excretion has to be prepared. High kidney retention of the peptides and antibody fragments - labelled with a metallic radionuclide - appeared to be one of the main obstacles in the potential use of small molecules in internal radiotherapy [3]. Therefore, we have developed a new chelator somatostatin analogue which can be labelled stably with the diagnostic radionuclide ${ }^{111} \mathrm{In}$ and its therapeutic chemical analogue ${ }^{90} \mathrm{Y}$. The new diagnostic radiotracer was studied in patients for its sensitivity and compared with the commercially available OctreoScan. In one patient, the somatostatin analogue labelled with the $\beta$-emitter ${ }^{90} \mathrm{Y}$ was tested for radionuclide therapy.

\section{Part 1: Diagnostic studies}

\section{Materials, patients and methods}

Radiotracer. We developed a new DOTA chelated somatostatin analogue, DOTA-D-Phe ${ }^{1}-$ Tyr $^{3}$-Octreotide (DOTATOC; DOTA: $1,4,7,10$-tetraazacyclododecane- $N, N^{\prime}, N^{\prime \prime}, N^{\prime \prime \prime}$-tetra-acetic acid) (Fig. 1), in a five-step synthetic procedure according to GMP

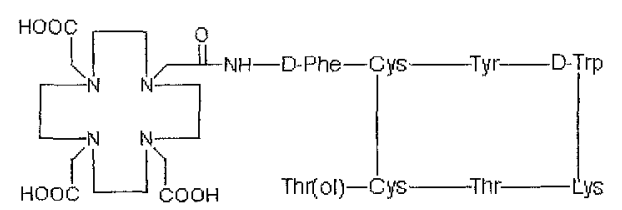

DOTATOC

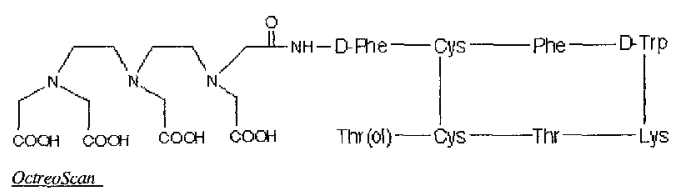

Fig. 1. Structure formulas of DOTATOC and OctreoScan 
practice. ${ }^{111}$ In-DOTATOC was prepared as follows: $8 \mu \mathrm{g}$ of DOTATOC were dissolved in $190 \mu 10.4 \mathrm{M}$ sodium acetate buffer (pH 5.5) with $7 \mathrm{mg}$ gentisic acid; after the addition of $6 \mathrm{mCi}$ ${ }^{11} \mathrm{InCl}_{3}(0.05 \mathrm{M} \mathrm{HCl}$, Mallinckrodt Med., Petten, The Netherlands), the solution was heated at $90^{\circ} \mathrm{C}$ for $25 \mathrm{~min}$. Quality control was obtained with the use of a Sep-Pak $\mathrm{C}_{18}$ cartridge and high-performance liquid chromatography (HPLC), resulting in highly pure radioligands with preserved receptor binding affinity $\left(K_{\mathrm{D}}=2.2 \pm 0.5 \mathrm{nM}\right)$.

Patients. Three patients with histopathologically verified neuroendocrine abdominal tumours were investigated after intravenous injection of $5 \mathrm{mCi}{ }^{111} \mathrm{In}$-OctreoScan and, 2 weeks later, $5 \mathrm{mCi}{ }^{111} \mathrm{In}$ DOTATOC (specific activity: $1 \mathrm{Ci} / \mu \mathrm{mol}$ ). Patient 1 (male, aged 46 years) had a remaining local tumour and peritoneal carcinosis after hemicolectomy because of an obstructing carcinoid tumour of the terminal ileum with four of five ileocaecal nodular lymph node stations being affected with metastases. At the time of admission, 5-hydroxy-3-indolacetic acid and chromogranin-B were positive. Patient 2 (male, aged 63 years) had a remaining tumour of $3 \times 2 \times 2 \mathrm{~cm}$ after subtotal splenopancreatectomy because of a malignant insulin-producing pancreatic tumour in the corpus with an initial diameter of $13 \mathrm{~cm}$. Patient 3 (male, aged 43 years) had metastatic spread of a neuroendocrine tumour of unknown origin with multiple liver, abdominal and skeletal metastases (Figs. 2, 3). The patient had received two unsuccessful cycles of chemotherapy according to the PEI scheme [cisplatin (Ebewe) $20 \mathrm{mg} / \mathrm{m}^{2}$ i.v. days $1-5$, etoposide (Vepesid) $75 \mathrm{mg} / \mathrm{m}^{2}$ i.v. days $1-5$, ifosfamide (Holoxan) $1.2 \mathrm{~g} / \mathrm{m}^{2}$ i.v. days 1-5; PEI: platin-etoposide-ifosfamide]. In his past, the patient had undergone surgery with a partial resection of the left kidney due to a haemorrhagic cyst. Conventional computer tomography (CT) of the abdomen and thorax on the day of admission showed cystic degeneration of the left kidney, two large liver metastases in the segments II $(4.5 \times 4 \mathrm{~cm})$ and $\mathrm{V}(9 \times 7.5 \mathrm{~cm})$ and multiple abdominal metastases. CT did not reveal any intrapulmonary or mediastinal infiltrations. Skeletal scintigraphy on the day of admission detected multiple bone metastases in the first, second and fourth lumbar vertebrae, in the third, seventh and ninth thoracic vertebrae, in the sacrum, in the third rib on the left ventrolateral side and, additionally, in the posterior skull (Fig. 2). Neuron-specific enolase was positive $(20 \mu \mathrm{g} / \mathrm{l})$ on the day of admission.

Methods. Planar scintigraphic images were obtained with a largefield-of-view gamma camera (Siemens DIACAM), equipped with a medium-energy parallel-hole collimator (matrix $64 \times 64$, zoom 1). The pulse height analyser windows were centered over both IIIIn photon peaks ( 172 and $246 \mathrm{keV}$ ) with a window width of $20 \%$. Data from both windows were then added to the acquisition frames. For the first $60 \mathrm{~min}$, dynamic images were acquired from posterior views of the abdominal region with 240 frames (15 s/frame); one image consisted of four summed dynamic frames. Static images ( $5 \mathrm{~min} /$ frame) were acquired from anterior and posterior views of the abdominal region $1,3,4.5,6,24$ and $48 \mathrm{~h}$ p.i.; in addition, static images from anterior views of the thoracic region and skull were acquired 24 and $48 \mathrm{~h}$ p.i. in patient 3. One image consisted of one static frame. Between the 4- and 24-h imaging and between the 24- and 48-h imaging, all patients were treated with Prontolax ( $10 \mathrm{mg}$ bisacodylum). Region of interest (ROI) analysis (in counts/pixel) of the background, kidneys, liver, tumour and/or metastases and spleen was obtained over the whole acquisition time. Within each patient, the ROI template was copied from one time frame to the next for intra-individual standardization. Each ROI was normalized to the uptake (in counts/pixel)

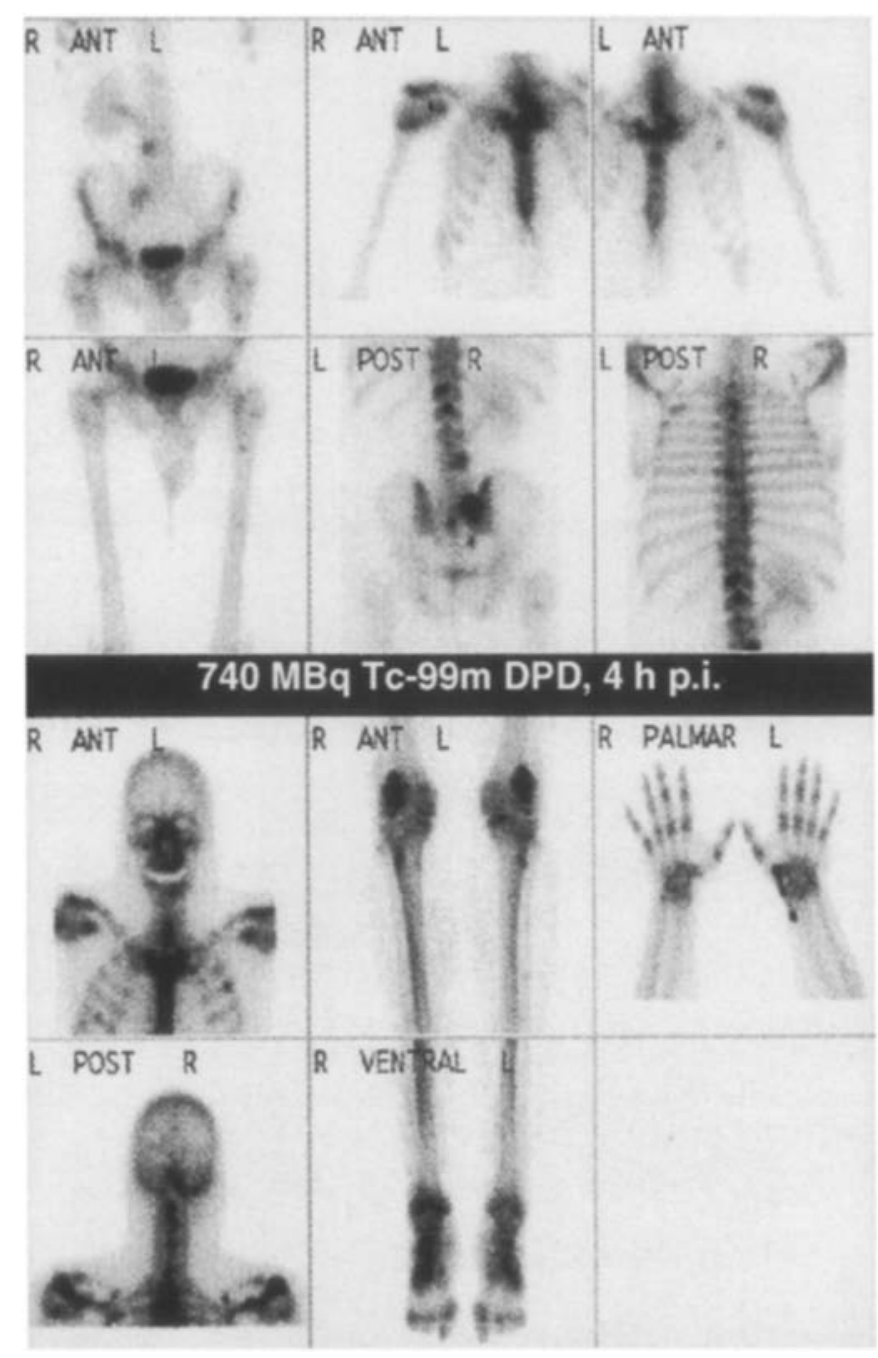

Fig. 2. Skeletal scintigraphy of patient 3 at the time of admission. $740 \mathrm{MBq} 99 \mathrm{~m}$ Tc-dicarboxydiphosphonate (DPD) was administered. Note the bone metastases in the sacrum, spine, rib and skull

of the summed time frames over $5 \mathrm{~min}$. All data were stored on a Siemens ICON computer system.

Radioactivity was measured in blood and urine over $48 \mathrm{~h}$. Blood samples were obtained after 2, 5, 10, 20 and $40 \mathrm{~min}$ and 1, 2, 3, 6, 24 and $48 \mathrm{~h}$. Urine was collected at 6 -h intervals over $48 \mathrm{~h}$. The chemical structure of the radioligand in blood and urine was determined by HPLC.

\section{Results}

Radioactivity cleared quickly from the blood. The blood clearance curve was fitted by two exponentials $(\alpha, \beta)$ obtaining half-times of $t_{1 / 2}(\alpha)=5 \pm 1 \mathrm{~min}(76 \%$ of radioactivity) and $t_{1 / 2}(\beta)=110 \pm 30 \mathrm{~min}$ ( $24 \%$ of radioactivity) (Fig. 4). The radiolabelled peptide showed high stability in vivo. No breakdown products were observed up to $6 \mathrm{~h}$ of observation. The radioactivity was mainly excreted via the kidney and found intact in the urine within the first $4 \mathrm{~h}$ ( $>96 \%$ intact, $>50 \%$ of activity in the urine). 

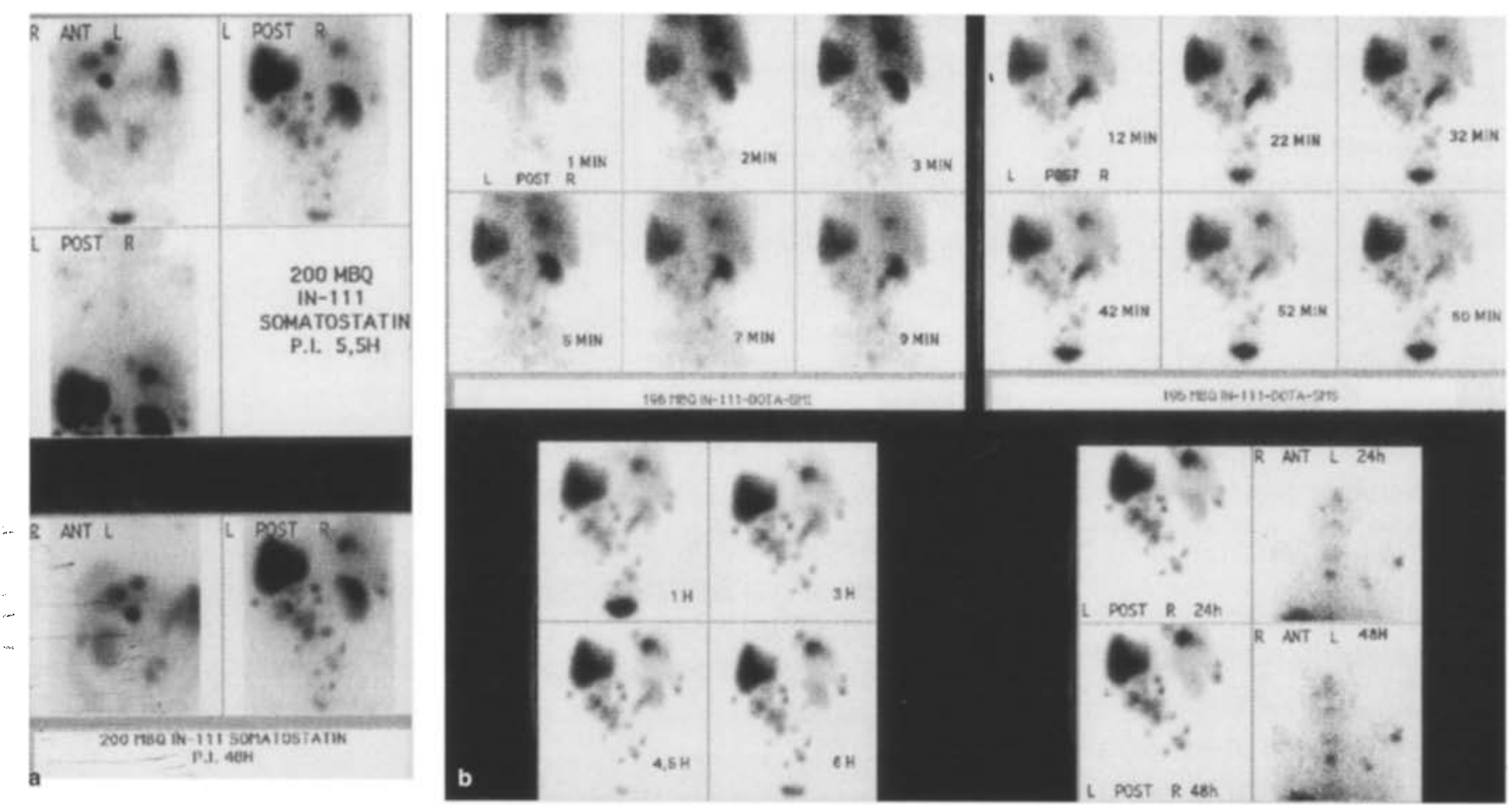

Fig. 3. a Scintiscans of patient $35.5 \mathrm{~h}$ and $48 \mathrm{~h}$ after intravenous injection of $200 \mathrm{MBq}{ }^{11}$ In-DTPA-D-Phe'-Octreotide (OctreoScan). Pretreatment scan: Note the high kidney uptake over the entire investigated time. The left kidney is without function due to cystic degeneration. b Scintiscans of patient 3 1-60 min and 1, 3, 4.5, 6, 24 and $48 \mathrm{~h}$ after intravenous injection of $196 \mathrm{MBq}$ of the newly developed substance ${ }^{11}$ In-DOTA-D-Phe ${ }^{1}-$ Tyr $^{3}$-Octreotide (DOTATOC). Pre-treatment scan 2 weeks later than the scan in a. Note the high kidney excretion within the first $12 \mathrm{~min}$ in contrast to the OctreoScan in a

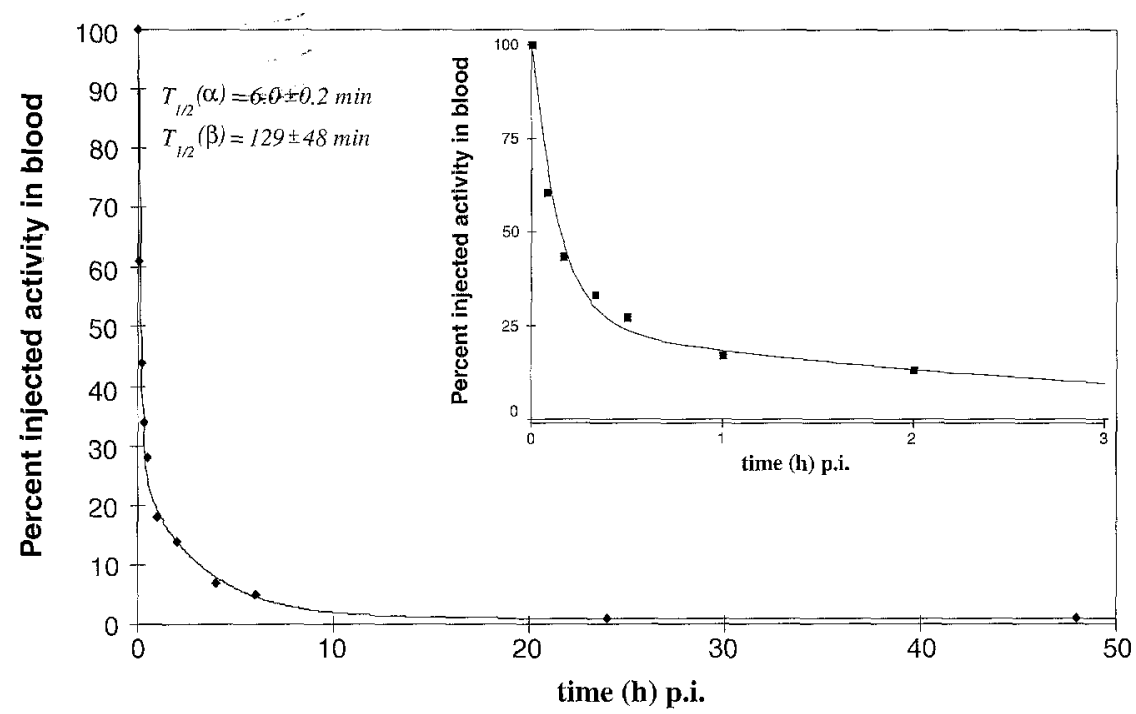

Fig. 4. Typical example of the blood clearance curve of ${ }^{11}$ In-DOTATOC (patient 1). The curve was fitted by two exponentials $(\alpha, \beta)$

Table 1. Uptake ratios 24 h after injection

\begin{tabular}{lllll}
\hline Radiotracer & patient & $\mathrm{k} / \mathrm{t}$ & $\mathrm{k} / \mathrm{a}$ & $\mathrm{t} / \mathrm{a}$ \\
\hline DOTATOC & 1 & 1.10 & 1.19 & 1.08 \\
OctreoScan & & 2.00 & 1.28 & 0.64 \\
DOTATOC & 2 & 1.33 & 2.68 & 2.02 \\
OctreoScan & & 2.54 & 5.80 & 2.28 \\
DOTATOC & 3 & 0.37 & 2.69 & 7.34 \\
OctreoScan & & 0.70 & 5.11 & 7.30 \\
\hline
\end{tabular}

k, Kidney; t, tumor; b, background; 1 , liver; a, injected activity (in $\mathrm{MBq}) / \mathrm{MBq}$
Two metabolites were detected in the urine at later times, the main one being [ $\left.{ }^{111} \mathrm{In}\right]\left(\right.$ DOTA-D-Phe ${ }^{1}$ ).

In all three patients, "11'In-DOTATOC showed the same diagnostic precision as OctreoScan, but superior biodistribution and faster blood and background clearance (Fig. 3). The kidney-to-tumour (k/t) uptake ratio (in counts per pixel) was on average 1.9-fold lower with 111 In-DOTATOC than with OctreoScan (Table 1). 


\section{Part 2: Treatment}

\section{Materials and methods}

Radiotracer: As a therapeutic radionuclide the pure $\beta$-emitter ${ }^{90} \mathrm{Y}$ was chosen. The labelling protocol was as described for ${ }^{11}$ In in Part 1 with the exception that different amounts of activity ( $25 \mathrm{mCi}$ and $40 \mathrm{mCi}$, respectively) were used, resulting in a highly pure and stable radioligand with preserved receptor binding affinity $\left(K_{\mathrm{D}}=2.6 \pm 0.5 \mathrm{nM}\right)$.

Patients. Due to the rapid progression of metastatic spread despite two courses of chemotherapy and persisting pain in the lower back and abdomen in patient 3 (for a detailed description of the patient's status, see Part 1), we decided to treat this patient experimentally by internal radiotherapy. Before treatment, tumour dosimetry was assessed. As the limiting factor for therapy was the kidney dose, the kidney retention was estimated by the use of a kidney phantom according to the patient's anatomical setting. Hereby, it was calculated that the kidney would receive a dose of $20 \mathrm{~Gy}$ if approximately $80 \mathrm{mCi}{ }^{90}$ Y-DOTATOC were applied (detailed data on tumour dosimetry are not presented in this short communication, but can be provided by the author). Therefore, we started fractionated treatment with two small portions each of $25 \mathrm{mCi}{ }^{90}$ Y-DOTATOC and one portion of $40 \mathrm{mCi}{ }^{90} \mathrm{Y}$-DOTATOC within 4 months (first treatment session: 21 October 1996; second session: 10 December 1996; third session: 3 February 1997). The treatment was approved by the Ethical Committee of the University of Basel.

Methods. In each ${ }^{90} \mathrm{Y}$-DOTATOC treatment session, $1 \mathrm{mCi}$ of ${ }^{11}$ In-DOTATOC was injected simultaneously in order to control the DOTATOC binding. Therefore, $1,4.5,24$ and $48 \mathrm{~h}$ p.i. static images ( $5 \mathrm{~min} / \mathrm{image}$ ) were acquired. In addition, 4 months after the last internal radiotherapy a follow-up ${ }^{111}$ In-DOTATOC scan was performed according to the protocol described in Part 1.

\section{Results}

During the 2 months following the last internal radiotherapy session in patient 3 , rapid tumour progression was stopped. This was verified by the follow-up ${ }^{111}$ InDOTATOC scan, which exhibited no further metastases and no growth of the known tumour masses. In addition, the tumour-to-background ratio of the metastases did not differ from that before treatment (data not presented). Furthermore, the only positive tumour marker, neuronspecific enolase, decreased from $20 \mu \mathrm{g} / \mathrm{l}$ to $<10 \mu \mathrm{g} / \mathrm{l}$ during this time. The patient's clinical status revealed a clear subjective improvement after therapy; in particular, the pain in his lower back and abdomen disappeared.

\section{Conclusion}

DOTATOC, labelled with ${ }^{11} \mathrm{In}$, is not only a possible new diagnostic agent but could, given its superior biokinetics and especially kidney-to-tumour uptake ratio, represent a new therapeutic alternative for somatostatin receptor-positive tumours and metastases when labelled with a $\beta$-emitter like ${ }^{90} \mathrm{Y}$. Further studies in humans with ${ }^{90} \mathrm{Y}$ are in progress.

Acknowledgements. The study was partially supported by the Swiss National Science Foundation (31-42516/94) and the "Regionale Krebsliga". We are further indebted to the radiotechnicians B. Leu, L. Schwob, V. Tschanz, T. Böhler and G. Zeh for their kind help.

\section{References}

1. Krenning EP, Bakker WH, Breeman WAP, et al. Localisation of endocrine-related tumours with radioiodinated analogue of somatostatin. Lancet 1989; I: 242-244.

2. Lamberts SWJ, Bakker WH, Reubi JC, Krenning EP. Somatostatin-receptor imaging in the localization of endocrine tumours. N Engl J Med 1990; 323: 1246-1249.

3. Sivolapenko GB, Douli V, Pectasides D, et al. Breast cancer imaging with radiolabelled peptide from complementarity-determining region of antitumour antibody. Lancet 1995; 346: $1662-1666$. 